\title{
Role of Mulberry Leaves in Human Nutrition: A Review
}

\section{Fareed Afzal ${ }^{1}$, Waseem Khalid ${ }^{1 *}$, Muhammad Naveed Asif ${ }^{1}$, Ayesha Jabeen ${ }^{1}$, Ravi Prakash Jha ${ }^{2}$, Muhammad Zubair Khalid ${ }^{1}$, Chasheen Fizza $^{1}$, Afifa Aziz' ${ }^{1}$, Ramish Akram ${ }^{3}$, Aqsa Bashir ${ }^{4}$, Sahar Younas ${ }^{4}$, Farwa Nayyer ${ }^{1}$, Rehan Yasin ${ }^{1}$ and Muhammad Zeeshan Ahmad ${ }^{1}$}

\author{
${ }^{1}$ Department of Food Science, Government College University, Faisalabad, Pakistan \\ ${ }^{2}$ Department of Community Medicine, Institute of Medical Sciences, Banaras Hindu \\ University, Varanasi, India \\ ${ }^{3}$ Department of Rehabilitation Sciences, The University of Faisalabad, Pakistan \\ ${ }^{4}$ National Institute of Food Science and Technology, University of Agriculture, \\ Faisalabad, Pakistan
}

*Corresponding Author: Waseem Khalid, Department of Food Science,

Government College University Faisalabad, Pakistan.

E-mail: waseemkhalid@gcuf.edu.pk
Received: February 05, 2021

Published: February 26, 2021

(C) All rights are reserved by Waseem Khalid., et al.

\begin{abstract}
Diabetes and different cardiovascular diseases are major problems across the globe for the human. Different traditional and modern medicinal techniques are being in use to cure these problems. Use of herbal medicines is one of them. Present review was conducted to determine the effects of mulberry plant powder for the treatment of these diseases. Mulberry (Morus alba L.) is important medicinal plant that contains numerous bioactive compounds including antioxidants i.e. flavonoids, phenolics as well as dietary fiber. Owing to presence of bioactive components, it shows strong effect against maladies like diabetes, cardiovascular disease and viral activities. Mulberry leaf powder is characterized for physicochemical characteristics (moisture content, pH, acidity, TSS, TPC). Moreover, due to high nutrients concentration, mulberry leaves are considered as vital for health.
\end{abstract}

Keywords: Mulberry Leaves; Phenolic Compounds; Bioactive Compounds; Diabetes; Cancer

\section{Introduction}

Herbs have quite a crucial part to play in the rehabilitation of various diseases worldwide. A structured action plan for improving food as a medicine [1], including identification of conventional medicine and presentation of a proper strategy and plan has recently been published by the WHO. In order to improve appropriate understanding of herbal medicines and the development of awareness particularly at university level is important. Development of herbal medicine cultivation is required and the establish- ment of a connection between traditional and modern treatment techniques. In that comprehensive action plan released by the World Health Organization, proper nutrition is strictly defined with respect to its medicinal sources [2].

Public interest in the functional food and nutraceuticals has been increased in the form of extracts particularly in herbal medicines. About $70 \%$ of germ doctors recommended herbal medicines for the treatment of various diseases that are widely used to treat mild to moderate depression than any chemical medication. Pho- 
to therapy is recommended reasonably safe because it includes many chemicals of a particular structure that act as an agent that improves effects or neutralize side effects. Scientific data for this presumption is restricted [3].

The mulberry plants with a crown stature of 5-6 feet from ground level and a stem bigness of 4-5 inches or more are called mulberry tree. They are incredibly developed by any of the combinations endorsed for rain-supported regions such as S-13 (for red loamy soil) or S-34 (dim cotton soil) that are resistant to drought or soil-moisture push conditions with the aid of all around produced saplings 8-10 months old. Every year in the middle of the rainstorm season (July-August) to a height of 5-6 feet and permitted to produce at the crown with the largest of 8-10 shoots. The leaves are collected three to four times a year, depending on the thunderstorm, by a leaf-picking process under rain-supported or semi-arid conditions. Mulberry has been made for quite some time in different nations with the sole purpose behind promoting the monophagous silkworm Bombyx mori L. It is used for many incredible purposes, for which it is called Kalpavriksha, regardless of the honest use of Mulberry Leaves as a silkworm engagement to goodness. The cutting-edge productivity on the way forward and the use of mulberry for the maintenance and medicinal use of creatures has been started by virtue of the yield for elective occupations of mulberry once the sericulture has rotted in the face of rivalries from outside nations when Japan and Italy should have an opportunity [4].

Mulberry (Morus alba) is a crucial helpful plant containing a number of bioactive compounds comprising cancer prevention agents included flavonoids, phenolics and dietary filaments in comparison. Attributable to the presence of bioactive components, it demonstrates a solid effect on disease such as diabetes, cardiovascular infection as well as viral exercises. Mulberry (Morus alba) is a profoundly useful plant that produces a range of bioactive compounds namely flavonoid, phenolic and dietary fibres specialists. It is inferable from the proximity of bioactive portions and has a powerful impact on ailments such as diabetes, cardiovascular infirmity and viral activity. Mulberry (Morus alba) explores the comprehensive grouping of critical pharmacological activities included antidiuretic, antimicrobial, antimutagenic, developmental neutralizing activity pro, anticancer, anxiolytic, anthelmintic, antistress, immunomodulatory, hypocholesterolemic, anticholeestrolmic and particular effects such as adaptons, hyperlipidemia, melanin biosynthesis containment. This plant also displayed antiatherogenic growth and the HIV activity was unfriendly [5].

According to the survey conducted by World Health organization, there are almost $80 \%$ developing countries whose population believed on the natural medicines which assure the safety of patients and improve the knowledge and skills of medicine provider for health safety. About $25 \%$ medicines are related to plants directly and indirectly [6]. Owing to their incapability to foundation side effects and combat antibiotic resistant microorganism plants are used as medicines [7]. About $80 \%$ of the population in rural areas rest on wild plants to meet the World Health Organization needs for the primary health worldwide [8]. Contrary to this, there are many types of plants which are the main pillar in traditional health care system and are used as medicine [9]. According to the pharmacological reviews the medicinal plants are potential source for the major antioxidants and bioactive compounds. Phytochemicals extracted from medicinal plants are elements for neutraceuticals [10]. There are about 6000 species of plants which represent 22 families and 150 genera in Pakistan. About $80 \%$ populations of rural areas of Pakistan depend on the herbal medicines [11].

In Pakistan about 400 species are widespread from 6000 which are $7.8 \%$. Due to availability and functionality of medicinal plants these are used in different areas of the world especially for respiratory disorder (10 species), diarrhea (12 species), urinary tract infection (UTI) Asthma (9 species), kidney stone (13 species) and rheumatism (6 species) [12]. There are 3000 species of plants which are reported from Gilgit Pakistan and from which almost 124 have medicinal values. Many studies have been conducted on the uses of Mulberry leaves to control the symptoms of medical condition. Some of the results of studies showed that Mulberry leaves can be used as protein source for ruminants. In sericulture industry Mulberry plant is used for determining the profitability and productivity. In sericulture industry the leaves of Mulberry are major component because quality of leaf produced per unit area has a direct bearing cocoon harvest. The maximization of mulberry leaf yield per unit area will lead to the realization of two most important objectives namely increased cocoon production per hectare and reduced cost of production [13]. The cultivation of Mulberry is an applied science involving detailed study of ecological, morphological and physiological features. The study comprises 
of cultivation techniques for the best Mulberry leaves production suited for rearing of silkworms and to obtain such leaves from a constant cultivation area in a reasonably inexpensive manner [14].

\section{Composition of mulberry}

Vitamins

Mulberry, which is rich in vitamins $\mathrm{B}$ and $\mathrm{C}$, can be used by systems for healthy, oxidizing and metabolic turnover of fats and starches for normal or accelerated activities. Similarly, the mulberry leaf contains rutin for dynamic hair-like structure, GABA for heartbeat reduction and DNJ for glucose reduction. In addition, sitosterol is used in mulberry leaves to reduce blood cholesterol levels. In later age, they found successful in relation to last experimental results with mice mulberry leaves. Mulberry leaf powder is now available as tablets or holders in the Korean market. Next emphasis in the Korean market are different techniques for the use of mulberry in humans [15].

Mulberry leaves are viable in directing fat while boosting digestion and are brimming with Vitamin C, A and B group of vitamins. It is highly safe to drink and immaculate non-lethal herb. It is useful for elderly to drink daily and can fight infection. Youthful kids, pregnant ladies and bosom bolstering moms should not drink substantial sums unless it is not recommended by qualified proficient [16].

\section{Bioactive components}

Many bioactive components are present in Mulberry leaves and fruits like anthocyanins, alkaloids and flavonoids [17]. Alkaloids are present in large concentration in Mulberry leaves included 1deoxynojirimycin (DNJ) which is the most potent glycosidase inhibitor that decreases the level of blood-sugar [18,19]. Mulberry have Resveratrol (trans-3,4 1,5-trihydroxystilbene) and Oxyresveratrol (trans-2,31,4,51tetrahydroxystilbene) which are hydroxystilbenes [20]. This bioactive substance has cardio protective and neuroprotection effect [21]. Oxyresveratrol has an inhibitory effect on tyrosinase to lessen the biosynthesis of melanin, used as cosmetic material and medical agent for disorders included hyper pigmentation [22]. Anthocyanins are a group of phenolic compounds naturally responsible for coloring of flowers, fruits and leaves. They are the best source of health benefits as anti-inflammatory and antioxidant compounds [23].
The extract of Mulberry anthocyanin has antimetastatis activity to inhibit migration of B16-F1 cells and anthocyanin has high inhibitory ability on lipid oxidation [24]. Flavonoids are commonly occurred in plant kingdom and mulberry found to contain at least four flavonoids including rutin. Flavonoids have been recognized to possess antioxidant, anti-inflammatory, antithrombotic, antiallergic, antiviral, hepatoprotective, and carcinogenic activities in human beings [25]. Plants are also rich in nutritive compounds like crude fat, crude protein, total sugars and mineral elements and are evaluated to get the correlation between the active components and their antioxidant activities.

\section{Alkaloids}

Aegelin, fragrine (C13H1103N), agelenine, marmeline, dictamine, N-2-hydrooxy-2(4-hydroxyphenyle) ethyle cinnamide, $\mathrm{N}$-2-[4-(3,3-dimethyleollyloxy) phenyl] ethyle cinnamide, $\mathrm{N}-2$ ethoxy-(2-methoxyphenyle) ethyle cinnamide, 0-methylehalfordinine, $\mathrm{N}$-4-methyleoxystyrl cinnamide, O-isopentenylehalfordinol, $\mathrm{N}$ 2-methoxy-2-[4-(3,3 dimethylallyloxy) phenyl] ethyle cinnamide, N-2-methoxy-(4-methoxyphenyle) ethylecinnamide and 0-(3,3 dimethyleallyle) halofordinol (25). Marmesin, Marmelosin, marmin, alloimperatorin, imperatorin, xanthotoxol, scoparone, methyl ether, scopaletin, umbelliferone, psoralen and marmelide, a-7 geranyleoxycoumarin and marmenole [26].

\section{Phenolic compounds}

Epidemiological examinations have confirmed that cancer prevention agent and contamination protective part and movement of the phenolics and high point many report varieties in phenolic compound as a component of plant species. Remarkable dissimilarities were to be observed the leaves of three mulberry collections for add up to phenolic content. These results noticeably advanced than publicized oil palm leaves [27], and Iranian restorative plant [28].

Anthocyanin is rich in mulberry extract and it is a colorant constituent naturally present in plant [29]. Anthocyanins indicated the antioxidant activity by scavenging the peroxyl radicals in trapping reaction. Anthocyanin glycosides after 8 hours of administration were no detected in GI tract. Sodium-dependent glucose transporter was used to transport anthocyanins across the erythrocyte. The bioactive constituents which are isolated from Morus alba like Kuwanon-G and leachianone showed antibacterial activities and 
1-deoxynojirimycin (DNJ) showed $\alpha$ glycosidase inhibitors activity. Similarly extract of Morus alba and its other compounds like flavonoids have antioxidant properties by protecting many organs from oxidative stress and scavenging free radicals [30].

\section{Medicinal properties of mulberry}

Mulberry leaves contain many pharmacological activities like reduction of hypertension, blood glucose, bacteriostatic, antivirus and antihyperlipidemic because they have amino acids, vitamins, quercetin, sugars, microelements and volatile oils. Lifespan can be intensified due to the presence of bioactive compounds in some species of mulberry [31]. Many other biochemical compounds can be isolated from mulberry plant like Kuwanol, Albafuran, Hydroxymoricin, Albanol, Morusin, Moranoline and Calystegine as they play an imperative role in the pharmaceutical industry, viewed by many scientists [32,33]. Profitable medicinal value can be obtained by medicinal properties of mulberry plant so pharmaceutical industry and research are attracted towards them. In herbal medicine, many conditions are treated using roots, stems, leaves, flowers, bark and fruits. There are many different names of mulberry herbal supplement and each of them invoke to products obtained from mulberry leaves having similar benefits and risks. Some names are no, wild pine, noni, mengkudu and caribe [34].

\section{Oxidative stress}

The imbalance between antioxidants and pro-oxidants mechanisms which results redundant oxidative metabolism. Several environmental factors such as medication, infection, radiations, exposure to pollutants, toxins, alcohol and poor diet etc. can contribute to this stress. It may lead to immense range of human diseases as it damages DNA, protein and other macromolecules which can cause cancer or heart diseases. Morus alba leaves has been used as food supplement in patients with certain diseases in that, the oxidative stress induced cellular injury in pathologically involved rats [35].

\section{Hypolipidemic activities}

Due to familiarity of its curative functions, Morus alba customarily utilized as hypolipidemic agent. Ficus mysorensis extracts Ethanol and hexane were examined and evaluated in rats suffering from hypercholesterolemia. Six fractions as well as Hexane were isolated and showed hypolipidemic activities [36]. The root bark of Morus alba L. (70\% alcohol extract) may behave as powerful hypocholesterolemic element plus inhibit the LDL anthrogenic alteration and limit lipidperoxidase synthesis in hypocholesterolemic mouse [37].

\section{Anti-inflammatory properties of mulberry}

With significant anti-inflammatory action a flavonoid, morin is found in mulberry. It was scrutinized that the accumulation of CsA in tissues of lymph and non-lymphoid tissues plus the in the immune cells of rats, is greatly influenced by morin. The more is morin intake the lesser is CsA level in tissues [38]. Flavonoid isolates of Morus nigra plus Morus alba bark are powerful anti-inflammatory agents [39].

\section{Atherosclerosis effects of mulberry}

In a study polyphenols viz quercetin (11.70\%), gallocatectin gallate (11.02\%) plus naringenin (9.012) are found to be present in leaf extracts of mulberry and polyphenols with their strong antiatherogenic activities. Both of the extracts restricted the peroxidation of lipids and oxidation of low density lipids where extracts of polyphenols tend to be more strong. Polyphenolic leaves extracts of Mulberry can be enhanced as anti-anther genic elements. Growth and migration of cells of vascular smooth muscles is known as Atherosclerosis. Mulberry leaf group displayed 40\% lessening in atherosclerotic size of lesion with comparison to that of control [40].

\section{Effect of mulberry in neurological disorders}

The shielding function of mulberry fruit extracts ethanol up to $70 \%$ in opposite to neurotoxicity inside and outside of the body of Parkinson"s disease models were investigated. Ultimately, mulberry considerably shielded the cells from neurotoxicity in a manner dependent upon doses [41].

\section{Effect of mulberry in neurological disorders}

The healthy influence of mulberry extract (ME) good in amounts of phenolics plus anthocyanins were studied. For 12 weeks snescent- accelerated mice [SAMP 8] and [SAMR1] of six months were fed with the diet supplement of $0.18 \%$ and $0.9 \%$ mulberry extracts. Those supplemented fed rats showed considerable reduction in amyloid beta protein plus improvement in memory to those mice which were treated with ME (Mulberry Extract), exhibited higher activity of antioxidant enzyme whereas less oxidation of lipid liver and brain in contrast to control rat [42].

Role of mulberry in cancer

Isolates of the extracts of ethyl acetate from roots of morus alba, Prenylated flavanone, 7, 2', 4',6'-tetrahydoroxy-6-geranylflavanone 
came to function as cytotoxic activists against cells of hepatoma in mice by7 the dose of an IC50 of $52.8 \mathrm{mg} / \mathrm{mL}$ [43]. Likewise, anthocyanins isolate of the fruit of Morus alba represented restricted effect on migration as well as invasion of highly metastatic A549 carcinoma cells of human lung [44]. The production of NO was inhibited and the tumor necrosis factor-a LPS activated RAW2647 was noticeably reduced by the methanolic extract plus its sub fractions obtained from aqueous butanol and chloroform fractions.

Mulberry leaves used in Japan and korea for different kind disease and mostly used in diabetes millatus and mostly used as anutraceutical and likewise utilized as a tea cake and noodle [45]. In this leaves flavonoids have been detected which is used as cancer inhibition profile agent and additional various parts of species observed [46]. Many cancer inhibition agent inspections have verified which neuroprotective, hypolipidemic, aggressive to hyperglycemic, antiviral, smoothing, cytotoxic exercise of various type of Mulberry and against hypotensive and HIV [47]. Soil cell support properties of Mulberry leaves, have specified interruption of oxidative peeling of oil and rice grain oil was seen to be disticnt better than the synthetic cancer prevention mediator. A famous element the numerous phytochemical ingredient in Mulberry leaves have practiced in few reports [48].

\section{Importance of mulberry in diabetics}

Chinese medicine used leaves of mulberry for cure of diabetes since a long ago due to presence of chemical compounds that reduce high blood sugar levels (hyperglycemia) [49]. After the intake of high sugar foods such as potatoes or sweets the blood sugar level rises sharply that stimulates the secretion of insulin to overcome the problem. But if requirement for more and more insulin happens much strongly with frequent times, the pancreatic ability of producing enough insulin diminish plus body cells become resistant to insulin because of its duty of facilitating transport of glucose across the cell walls. Thus this resistance to insulin being dangerous is the cause of type 2 diabetes. Primarily caused by obesity. Generally, if a man is obese, the risk of diabetes is greater. It was found by a Japanese research group, that the leaves of white Mulberry exhibit compounds that inhibit these intestinal enzymes. Mulberry have 1-deoxynojirimycin (DNJ) plus its derivatives such as alpha-glucosidase inhibitors that are utilized as curatives for diabetes mellitus [50].
Phytochemical-rich plants play a crucial role in consuming less calorie-based diabetes mellitus medicines. Mulberry contains 1-deoxynojirimycin (DNJ) and some of its dependents, which are alphaglycosidase inhibitors [51,52] used as drugs for the treatment of mellitus diabetes. Mixtures of alpha-glycosidase in the intestinal lumen and in the brush edge film have a fundamental role in the digestion of starch and oligosaccharides into monosaccharides until they are kept [5]. Covering the progress of such stomach-related forces would put off the deterioration of starch including oligosaccharides, thereby reducing glucose consumption and thus covering the stature of postprandial blood glucose levels [53]. Starting late, two or three trials in creatures and people have reported that DNJ-containing mulberry or sericulture stuff cover post-prandial glucose development $[18,51,52]$.

Mulberry leaves are a known usual solution in Asian countries, for prevention diabetes, previous report for example indicated. A strong GI stroke have Mulberry leaves on version of 1- deoxynojirimycin (DNJ) a glucose modest. Moreover, Mulberry leaves nutrition to anticipate diabetes we check the possibility of this concentrate for postprandial glycemic control through human trails went for moving utilization [54].

In this study microarray inspection was used DNA to discover expression quality in the liver of hypercholesterolemia mice preserved with rich polyphenol mulberry leaf explain the instrument concentrate to associated with hypercholesterolemia effect of the distillate. This search is to describe the principle goal of the mulberry leaves, physic-synthetic inspection of mulberry tea and the hyperglycaemic breakdown and mulberry takes off ability of hyperlipidaemia. Importance point of restorative Mulberry tea is the tea which is removed from mulberry tree takes off. Asian yet now it is distinctive to produce world over. White Mulberry and faint mulberry are the most known generous among the 10 generous. In pre-winter the leaves are collected and dehydrated for making tea [55].

\section{Conclusion}

The current review conclude that Mulberry leaves are played vital role in human health against several diseases. Mulberry leaves are composed of antioxidants, flavonoid, phenolic compounds and dietary fiber that are used as herbal medicines against cancer, diabetes and neurological disorders. 


\section{Bibliography}

1. Naseri M. "Traditional Iranian Medicine (Tim) and it's promotion with guidelines of world health organization". (2004): 5366.

2. Fallah Huseini H., et al. "Review of anti-diabetic medicinal plant used in traditional medicine". Journal of Medicinal Plants 1.17 (2006): 1-8.

3. Rafieian-Kopaei M., et al. "Plants antioxidants: From laboratory to clinic". Journal of Nephropathology 2.2 (2013).

4. Li Ke., et al. "Mulberry-like dual-drug complicated nanocarriers assembled with apogossypolone amphiphilic starch micelles and doxorubicin hyaluronic acid nanoparticles for tumor combination and targeted therapy". Biomaterials 39 (2015): 131-144.

5. Standl E and Oliver S. "Alpha-glucosidase inhibitors 2012-cardiovascular considerations and trial evaluation". Diabetes and Vascular Disease Research 9.3 (2012): 163-169.

6. Gragg GM., et al. "Natural products in drug discovery and development". Journal of Natural Products 60 (1997): 52.

7. Rawat RBS and Uniyal RC. "National Medicinal Plants Board, Committed for overall development of the sector". Agros Dictionary of Medicinal Plants 1 (2003): 12-16.

8. Alves Rômulo RN and Ierecê LR. "Why study the use of animal products in traditional medicines?". Journal of Ethnobiology and Ethnomedicine 1.1 (2005): 1-5.

9. Prusti A. "Antibacterial activity of some Indian medicinal plants". Ethnobotanical Leaflets 2008.1 (2008): 27.

10. Ncube NS., et al. "Assessment techniques of antimicrobial properties of natural compounds of plant origin: current methods and future trends". African Journal of Biotechnology 7.12 (2008).

11. Ahmed H. "Issues regarding the medicinal plants of Pakistan". Udyana Today 6 (1999): 6-7.

12. Khan RU., et al. "Weed flora of sugarcane in district Bannu, Khyber Pakhtunkhawa, Pakistan". Pakistan Journal of Weed Science Research 18.4 (2012).
13. Khurana P and Vibha GC. "The advent of genomics in mulberry and perspectives for productivity enhancement". Plant Cell Reports 30.5 (2011): 825-838.

14. Vijayan K. "Approaches for enhancing salt tolerance in mulberry (Morus L)-A review". Plant Omics 2.1 (2009): 41.

15. Lee CY., et al. "Mulberry leaves protect rat tissues from immobilization stress-induced inflammation". Biofactors 31.1 (2007): 25-33.

16. Yuan $Q$ and Longyan Z. "The Mulberry (Morus alba L.) Fruit? A Review of Characteristic Components and Health Benefits". Journal of Agricultural and Food Chemistry 65.48 (2017): 10383-10394.

17. Vijayan K. "Genetic relationships of Japanese and Indian mulberry (Morus spp.) genotypes revealed by DNA fingerprinting". Plant Systematics and Evolution 243.3 (2004): 221-232.

18. Kimura T., et al. "Food-grade mulberry powder enriched with 1-deoxynojirimycin suppresses the elevation of postprandial blood glucose in humans". Journal of agricultural and food chemistry 55.14 (2007): 5869-5874.

19. Nakagawa K., et al. "Determination of iminosugars in mulberry leaves and silkworms using hydrophilic interaction chromatography-tandem mass spectrometry". Analytical Biochemistry 404.2 (2010): 217-222.

20. Bae SH and Hyung-Joo S. "Antioxidant activities of five different mulberry cultivars in Korea". LWT-Food Science and Technology 40.6 (2007): 955-962.

21. Chao WW., et al. "The production of nitric oxide and prostaglandin E2 in peritoneal macrophages is inhibited by Andrographis paniculata, Angelica sinensis and Morus alba ethyl acetate fractions". Journal of Ethnopharmacology 122.1 (2009): 68-75.

22. Choi EM and Jae-Kwan H. "Effects of Morus alba leaf extract on the production of nitric oxide, prostaglandin E2 and cytokines in RAW264. 7 macrophages". Fitoterapia 76.7-8 (2005): 608613.

23. Nuengchamnong N., et al. "Quantitative determination of 1-deoxynojirimycin in mulberry leaves using liquid chromatogra- 
phy-tandem mass spectrometry". Journal of Pharmaceutical and Biomedical Analysis 44.4 (2007): 853-858.

24. Cui XQ., et al. "A new Diels-Alder type adduct and two new flavones from the stem bark of Morus yunanensis Koidz". Journal of Asian Natural Products Research 10.4 (2008): 315-318.

25. Pennathur S., et al. "Potent antioxidative activity of lycopene: A potential role in scavenging hypochlorous acid". Free Radical Biology and Medicine 49.2 (2010): 205-213.

26. Venkatesh Kumar R and Seema Chauhan. "Mulberry: life enhancer". Journal of Medicinal Plants Research 2.10 (2008): 271278.

27. Zhang M., et al. "In vivo hypoglycemic effects of phenolics from the root bark of Morus alba". Fitoterapia 80.8 (2009): 475-477.

28. Bouayed J., et al. "Chlorogenic acid, a polyphenol from Prunus domestica (Mirabelle), with coupled anxiolytic and antioxidant effects". Journal of the neurological sciences 262.1-2 (2007): 77-84.

29. Rossetto M., et al. "Peroxyl radical trapping activity of anthocyanins and generation of free radical intermediates". Free Radical Research 41.7 (2007): 854-859.

30. Kapche G., et al. "Prenylated arylbenzofuran derivatives from Morus mesozygia with antioxidant activity". Phytochemistry 70.2 (2009): 216-221.

31. Venkatesh Kumar R and Seema Chauhan. "Mulberry: life enhancer". Journal of Medicinal Plants Research 2.10 (2008): 271278.

32. Andallu B., et al. "Effect of mulberry (Morus indica L.) therapy on plasma and erythrocyte membrane lipids in patients with type 2 diabetes". Clinica Chimica Acta 314.1-2 (2001): 47-53.

33. Singhal B., et al. "Approaches to industrial exploitation of mulberry (mulberry sp.) fruits". Journal of Fruit and Ornamental Plant Research 18.18 (2010): 83-99.

34. Zeng Q., et al. "Introduction trial of medicine mulberry (Morus nigra) in Chongqing". Zhongguo Zhong yao za zhi= Zhongguo zhongyao zazhi= China journal of Chinese Materia Medica 41.8 (2016): 1450-1455.
35. Jaruchotikamol Atika and Patchareewan Pannangpetch. "Сytoprotective activity of mulberry leaf extract against oxidative stress-induced cellular injury in rats". Pakistan Journal of Pharmaceutical Sciences 26.1 (2013).

36. Ahmad A., et al. "Antiulcer and antioxidant activities of a new steroid from Morus alba”. Life Sciences 92.3 (2013): 202-210.

37. El-Beshbishy H., et al. "Hypolipidemic and antioxidant effects of Morus alba L. (Egyptian mulberry) root bark fractions supplementation in cholesterol-fed rats". Life Sciences 78.23 (2006): 2724-2733.

38. Yu Zhifeng., et al. "Morin (3, 5, 7, 2', 4'-pentahydroxyflavone) exhibits potent inhibitory actions on urate transport by the human urate anion transporter (hURAT1) expressed in human embryonic kidney cells". Drug Metabolism and Disposition 35.6 (2007): 981-986.

39. Wang L., et al. "Three new compounds from Morus nigra L. Original Article". Journal of Asian Natural Products Research 12.6 (2010): 431-437.

40. Harauma A., et al. "Mulberry leaf powder prevents atherosclerosis in apolipoprotein E-deficient mice". Biochemical and Biophysical Research Communications 358.3 (2007): 751-756.

41. Kim H., et al. "Mulberry fruit protects dopaminergic neurons in toxin-induced Parkinson's disease models". British Journal of Nutrition 104.1 (2010): 8-16.

42. Zhang W., et al. "HPLC-DAD-ESI-MS/MS analysis and antioxidant activities of nonanthocyanin phenolics in mulberry (Morus alba L.)". Journal of Food Science 73.6 (2008): C512C518.

43. Hisayoshi Kofujita., et al. "A novel cytotoxic prenylated flavonoid from the root of Morus alba". Journal of Insect Biotechnology and Sericology 73.3 (2004): 113-116.

44. Colonna M., et al. "Cancer prevalence in France: time trend, situation in 2002 and extrapolation to 2012". European Journal of Cancer 44.1 (2008): 115-122.

45. Kim H., et al. "Anti-oxidative capacity of mulberry leaf and its tea". Journal of Sericultural and Entomological Science 49.1 (2007): 18-23. 
46. Iqbal Shahid and M I Bhanger. "Effect of season and production location on antioxidant activity of Moringa oleifera leaves grown in Pakistan". Journal of food Composition and Analysis 19.6-7 (2006): 544-551.

47. Du J., et al. "Antiviral flavonoids from the root bark of Morus alba L". Phytochemistry 62.8 (2003): 1235-1238.

48. Li Y., et al. "Hybrid of 1-deoxynojirimycin and polysaccharide from mulberry leaves treat diabetes mellitus by activating PDX-1/insulin-1 signaling pathway and regulating the expression of glucokinase, phosphoenolpyruvate carboxykinase and glucose-6-phosphatase in alloxan-induced diabetic mice". Journal of Ethnopharmacology 134.3 (2011): 961-970.

49. Miyahara C., et al. "Inhibitory effects of mulberry leaf extract on postprandial hyperglycemia in normal rats". Journal of $\mathrm{Nu}$ tritional Science and Vitaminology 50.3 (2004): 161-164.

50. Mudra M., et al. "Influence of mulberry leaf extract on the blood glucose and breath hydrogen response to ingestion of 75 g sucrose by type 2 diabetic and control subjects". Diabetes Care 30.5 (2007): 1272-1274.

51. Asano N., et al. "Polyhydroxylated alkaloids isolated from mulberry trees (Morus alba L.) and silkworms (Bombyx mori L.)". Journal of Agricultural and Food Chemistry 49.9 (2001): 4208-4213.

52. Nakamura Mariko., et al. "Suppressive response of confections containing the extractive from leaves of Morus Alba on postprandial blood glucose and insulin in healthy human subjects". Nutrition and Metabolism 6.1 (2009): 1-10.

53. Derosa Giuseppe and Pamela Maffioli. "Efficacy and safety profile evaluation of acarbose alone and in association with other antidiabetic drugs: a systematic review". Clinical Therapeutics 34.6 (2012): 1221-1236.

54. Samkol Pok and S D Lukefahr. "A challenging role for organic rabbit production towards poverty alleviation in south east Asia”. Proc. 9th World Rabbit Congress, Verona, Italy. (2008).

55. Kobayashi Yukihiro., et al. "The use of Morus alba L. (mulberry) and Eucommia ulmoides (Tochu) leaves as functional foods: a promising approach in the management of hyperlipidemia". Journal of Traditional Medicines 27.5 (2010): 225-230.

\section{Assets from publication with us}

- Prompt Acknowledgement after receiving the article

- Thorough Double blinded peer review

- Rapid Publication

- Issue of Publication Certificate

- High visibility of your Published work

Website: www.actascientific.com/

Submit Article: www.actascientific.com/submission.php

Email us: editor@actascientific.com

Contact us: +919182824667 